\title{
A novel biomechanical analysis of gait changes in the MPTP mouse model of Parkinson's disease
}

Werner J Geldenhuys, Tamara Guseman, Ilse S. Pienaar, Dean E Dluzen, Jesse W Young

Parkinson's disease (PD) is an age-associated neurodegenerative disorder hallmarked by a loss of mesencephalic dopaminergic neurons. Accurate recapitulation of the PD movement phenotype in animal models of the disease is critical for understanding disease etiology and developing novel therapeutic treatments. However, most existing behavioral assays currently applied to such animal models fail to adequately detect and subsequently quantify the subtle changes associated with the progressive stages of PD. In this study, we used a video-based analysis system to develop and validate a novel protocol for tracking locomotor performance in the 1-methyl-4-phenyl-1,2,3,6-tetrahydropyridine (MPTP) mouse model of PD. We anticipated that 1 ) treated mice should use slower, shorter, and less frequent strides and 2) that gait deficits should monotonically increase following MPTP administration, as the effects of neurodegeneration become manifest. Video-based biomechanical analyses, utilizing behavioral measures motivated by the comparative biomechanics literature, were used to quantify gait dynamics over a seven-day period following MPTP treatment. Analyses revealed shuffling behaviors consistent with the gait symptoms of advanced PD in humans. Here we also document dramatic gender-based differences in locomotor performance during the progression of the MPTP-induced lesion, despite male and female mice showing similar losses of striatal dopaminergic cells following MPTP administration. Whereas female mice appeared to be protected against gait deficits, males showed multiple changes in gait kinematics, consistent with the loss of locomotor agility and stability. Overall, these data show that the novel video analysis protocol presented here is a robust method capable of detecting subtle changes in gait biomechanics in a mouse model of PD, indicating that this method is a useful means by which to easily and economically screen preclinical therapeutic compounds for protecting against or reversing neuropathology associated with PD neurodegeneration. 


\section{A novel biomechanical analysis of gait changes in the MPTP mouse model of Parkinson's disease}

3
Werner J. Geldenhuys ${ }^{1}$, Tamara Guseman ${ }^{2}$, Ilse S. Pienaar ${ }^{3}$, Dean E. Dluzen ${ }^{2 \#}$, Jesse Young²*

${ }^{1}$ Department of Pharmaceutical Sciences, College of Pharmacy, Northeast Ohio Medical

University (NEOMED), Rootstown, Ohio, USA

${ }^{2}$ Department of Anatomy and Neurobiology, College of Medicine, Northeast Ohio Medical University (NEOMED), Rootstown, Ohio, USA

${ }^{3}$ Center for Neurodegeneration and Neuroinflammation, Division of Brain Sciences, Department of Medicine, Imperial College London, London, United Kingdom

${ }^{\#}$ Current Address: Department of Anatomy, Southern Illinois University School of Medicine, Carbondale, Illinois, USA

SHORT TITLE: Gait Dynamics of Parkinsonian Mice

KEYWORDS: Behavior; Gait; Gender-bias; MPTP; Locomotion

* Corresponding author

Name: Jesse W. Young

Address: Department of Anatomy and Neurobiology

Northeast Ohio Medical University

4209 State Route 44

Rootstown, OH 44272

Phone: 1-330-325-6304

Email: jwyoung@neomed.edu 
Parkinson's disease (PD) is an age-related neurodegenerative disease, where individuals aged older than 60 years of age show increased risk of developing the disorder (Connolly \&

33 Lang 2014). A triad of classical motor symptoms is seen in advanced PD patients, consisting of 34 rigidity, akinesia, and tremor (DeLong \& Wichmann 2009). These symptoms appear following 35 the loss of at least $80 \%$ of the dopaminergic neurons within the Substantia Nigra pars compacta 36 (SNpc) (Hartmann 2004), thereby impairing a patient's ability to perform everyday tasks 37 (Aviles-Olmos et al. 2013). As the disease progresses, co-morbid non-motor symptoms manifest, including cognitive impairments and depression (Lawson et al. 2014; Obeso et al. 2014), which are often resistant to dopamine (DA) replacement therapies (Connolly \& Lang 2014). Currently available therapies are mainly aimed at replacing the lost striatal DA content. With dopaminergic cell loss continuing and the side-effects associated with synthetic DA replacement increasing, continuous use of pharmacotherapeutics fails to alter disease progression (Connolly \& Lang 2014). On the other hand, deep brain stimulation (DBS) therapy shows dramatic improvements in some late-stage PD patients, including improved gait and postural instability following DBS implanted in the pedunculopontine nucleus (PPN), arguing strongly for a case that patients might benefit substantially more, should intervention be initiated at an earlier stage of the disease progression (Mazzone et al. 2014). Related to this, recent work revealed that DBS targeting the subthalamic nucleus (STN) induces vascular remodeling effects, including an upregulation of the vascular endothelial growth factor (VEGF), suggesting that DBS induces plasticity-related effects (Pienaar et al. 2015). Hence, optimization of intervention protocols stand to benefit greatly from a reliable animal model of PD that mimics progressive stages of the disease, in line with the clinical aim of initiating treatment at an earlier stage during progressive PD. 
Accurate recapitulation of the movement phenotype seen in PD patients in animals is

54 important for the assessment and development of novel therapeutic treatments as well as for 55 providing a tool by which to gain insights into the molecular and cellular mechanisms 56 contributing to the loss of neurons and the concomitant circuit disruptions that characterize 57 human PD. In this regard, sensitive behavioral paradigms are of paramount importance for 58 characterizing existing and newly introduced genetic-based and toxin-induced animal models of 59 neurodegenerative disease (Bury \& Pienaar 2013; Pienaar et al. 2012). Unfortunately, many of 60 the behavioral assays applied to animal models fail to adequately detect the subtle changes 61 associated with the different stages of the disease (Antony et al. 2011; Meredith \& Rademacher 62 2011). In this study, we used a novel video-based paradigm for analyzing gait and locomotor 63 kinematics to detect the subtle longitudinal changes in locomotor performance occurring in the 64 methyl-4-phenyl-1,2,3,6-tetrahydropyridine (MPTP) mouse model of PD, and analyzed the 65 results in a gender-specific manner. Mice and non-human primates systemically injected with 66 MPTP, a mitochondrial neurotoxin, show loss of mesencephalic dopaminergic neurons with 67 concomitant loss of striatal DA content, resulting in motor deficits (Bezard \& Przedborski 2011; 68 Pienaar et al. 2012; Schmidt \& Ferger 2001). We anticipated that 1) MPTP-treated mice should 69 move at slower speeds and with shorter, less frequent strides, mimicking the bradykinesia shown 70 by PD patients (Fernagut et al. 2002) and that 2) gait deficits should monotonically increase 71 following MPTP administration, as the effects of neurodegeneration become manifest (Klemann 72 et al. 2015). Finally, we also tested for a gender effect in the locomotor response to MPTP 73 treatment, given that previous research has established sex-based differences in the phenotype of 74 both PD patients and rodent models of the disease (Antzoulatos et al. 2010; Gillies et al. 2014; 75 Van Den Eeden et al. 2003). 


\section{Study design}

8

Research was carried out at Northeast Ohio Medical University (NEOMED), in strict accordance with the recommendations in the Guide for the Care and Use of Laboratory Animals of the National Institutes of Health. All procedures were pre-approved by the NEOMED Institutional Animal Care and Use Committee (NEOMED IACUC Protocol 10-006).

Three cohorts of C57BL/6J laboratory mice $(n=4$ per group, each consisting of 2 males and 2 females) were used. One male mouse from the first cohort and one female mouse from the second cohort did not show the characteristic striatal DA depletion following MPTP treatment. Additionally, one female mouse from the third cohort lost a significant amount of body weight following MPTP treatment and was removed from the study. Therefore, our final sample consisted of nine mice (five males and four females). On average, male mice weighed more than the female mice (mean female body mass [95\% confidence limits]: $23.7 \mathrm{~g}$ [23.02 g, $24.32 \mathrm{~g}$ ], mean male body mass: $29.1 \mathrm{~g}$ [27.56 g, $30.66 \mathrm{~g}]$; measured prior to MPTP treatment).

The three cohorts were tested in chronological order, such that at the start of data collection, the mice in cohort 1 were 12 weeks of age, those in cohort 2 were 14 weeks of age, and those in cohort 3 were 16 weeks of age. Following a training period lasting from 3-4 days, during which mice were acclimated to the experimental procedure, each animal was evaluated in an initial behavioral assessment to quantify baseline locomotor performance. Following this baseline assessment, mice were treated with a single dose of MPTP (35 mg/kg; i.p.) and then evaluated for seven days of longitudinal locomotor testing, starting at the first day post treatment, but at least $24 \mathrm{~h}$ after MPTP treatment to bypass some of the most acute phenotypic effects due to the drug's toxicity (e.g., epilepsy-like symptoms: Klemann et al. 2015). In addition to the 
99 baseline evaluation, every mouse in the dataset was evaluated at the seventh day after treatment.

100 However, to improve the efficiency of our data collection and reduce the activity burden on the

101 mice, on the remaining days (i.e. days 1-6 following MPTP treatment) experiments were

102 staggered to ensure that we collected data from at least two cohorts of mice per experimental day

103 (i.e. cohort 1 was evaluated on days 1-4, cohort 2 on days $1,2,5,6$, and cohort 3 on days 3-7).

104 Mice were then euthanized via cervical dislocation, the striata removed and the DA levels 105 determined using high-pressure liquid chromatography (HPLC), described below.

106 We chose not to include a "sham" control group in our experimental design, but to rather

107 use mixed-model repeated measures statistical analyses (detailed below) to test for the gait-

108 related sequelae of MPTP treatment against each individual's pre-treatment baseline. This design

109 reduced the number of animals required for our study, satisfying ethical concerns relating to

110 reducing the number of animal subjects used for achieving robust statistical results. Our

111 statistical design was combined with an intensive data collection protocol (15-25 locomotor trials

112 collected per day, per experimental animal), enabling us to accurately characterize the efficacy of

113 our new method and obtain statistically significant results, despite a relatively small sample size.

114 Apparatus and locomotor testing

115 The mice were made to run along a white wooden trackway ( $41.75 \mathrm{~cm}$ long, $4 \mathrm{~cm}$ wide)

116 into a dark box located at the terminal end of the track. The trackway was placed on a laboratory

117 bench top, approximately $1 \mathrm{~m}$ off the ground. Mice were filmed with a high-speed digital camera

118 (MotionScope Model PCI 1000s, Redlake MASD Inc., USA) placed overhead to allow for a

119 dorsal view of the mouse running along the trackway. Videos were recorded at 200 frames per

120 second (fps) with a $1 / 2000$ shutter speed. The trackway was illuminated with a 250 -watt quartz

121 light (Lowel-Light Manufacturing Inc., USA) to provide adequate depth of field. On either side 
122 of the runway, mirrors were placed at $45^{\circ}$ angles to the sagittal plane to provide complete views

123 of the footfalls of each limb during locomotion (Fig. 1). We recorded 15-25 strides per mouse

124 per day. All testing took place between 10AM and 2PM, during the active (dark) phase of these

125 nocturnal animals.

126

127

128

129

130

131

132

133

134

135

136

138

140

141

142 point masses localized at the average static center of mass $(\mathrm{COM})$ position for each gender.

143 Measurements of static COM position in cadaveric male and female mice $(\mathrm{N}=3$ males and $\mathrm{N}=2$

144 females) using the reaction board method (Ozkaya \& Nordin 1999; Young 2012) showed some 
145 variation between genders, but little variation between individuals within genders (male mean

146 COM position: $41.5 \%$ of nose-to-tail base length, coefficient of variation: $6.9 \%$; female mean

147 COM position $45.5 \%$ of nose to tail length, coefficient of variation $3.1 \%$ ). The COM was

148 therefore defined as a point $41.5 \%$ along the length of the nose-to-tail base vector in males and

$14945.5 \%$ along the length of the nose-to-tail base vector in females.

150 Although our proxy is necessarily a simplification of actual COM position, which can be

151 expected to vary based on both body configuration during locomotion, variation in the effective

152 length of the trunk (i.e. the euclidean distance between the nose and the tail base) was minimal

153 during locomotor strides (average coefficient of variation with $95 \%$ confidence bounds, $4.08 \%$

$154[3.822 \%, 4.337 \%]$ in males, $3.81 \%[3.507 \%, 4.114 \%]$ in females). These data suggest that little

155 lateral flexion of the vertebral column had occurred and indicate that our approach of modeling

156 the trunk as a linear vector should have minimal effect on the accuracy of our COM estimates.

157 Raw data on footfall timings and modeled COM displacements were used to calculate

158 several metrics of locomotor performance. Average locomotor speed $(\mathrm{cm} / \mathrm{sec})$ was calculated as

159 the absolute value of mean velocity across the stride. Stride length $(\mathrm{cm})$ was defined as the net

160 distance travelled by the COM during the stride. Stride frequency $(\mathrm{Hz})$ was calculated as the

161 inverse of stride duration. Additionally, we calculated two metrics of overall postural stability.

162 First, based on the timings of limb touchdown and liftoff events, we calculated the percentage of

163 stride duration spent in various support combinations (e.g. supported by one, two, three or four

164 limbs). We then calculated the mean support number using the equation: \% single-limb support +

165 (2 $\times \%$ double-limb support $)+(3 \times \%$ triple-limb support $)+(4 \times \%$ quadruple-limb support $)$. A

166 higher mean support number indicates that a greater number of limbs provide support at any one

167 instance during the stride, theoretically conferring greater postural stability to the animal. 
168 Additionally, we calculated a "sway" index to quantify mediolateral stability, based on the

169 "straightness" index of Jamon and Clarac (Jamon \& Clarac 1998). The sway index was

170 computed as the ratio of the total horizontal path distance travelled by the COM during the stride

171 and the straight-line distance between the COM coordinates at the beginning and the end of the

172 stride, multiplied by 100 . A sway index of 100 indicates a perfectly linear path with higher

173 values indicating increasing amounts of mediolateral sway.

174

175

176

177

178

179

180

181

182

183

184

185

186

187

188

189

190

\section{$D A$ content analysis}

We measured remaining levels of DA following systemic MPTP treatment, as previously described (Geldenhuys et al. 2014). In brief, mice were euthanized via cervical dislocation, the brain was removed from the skull, and striatal tissue was dissected out then snap frozen in liquid nitrogen. For processing, the striatal tissue was weighed and placed in cold perchloric acid $(0.1 \mathrm{~N}$, $\left.500 \mu \mathrm{L}, 4^{\circ} \mathrm{C}\right)$. Tissue samples were sonicated and centrifuged. An aliquot was removed to measure DA levels within the bilateral striata across the various cohorts. Tissue samples were evaluated for DA content by means of HPLC coupled with electrochemical detection. Biogenic amines were separated on a Supelco column (Discovery C18, $10 \mathrm{~cm} \times 3 \mathrm{~mm} \times 5 \mu \mathrm{m}$ ). Samples were injected into a $20 \mu \mathrm{L}$ loop. A degassed isogradient mobile phase consisting of sodium acetate $(50 \mathrm{mM})$, citric acid $(27.4 \mathrm{mM})$, sodium hydroxide $(10 \mathrm{mM})$, sodium octyl sulfate $(0.1$ $\mathrm{mM})$, ethylenediaminetetraacetic acid (EDTA) $(0.1 \mathrm{mM})$ and $5 \%$ methanol in filtered deionized water was used for the system. The mobile phase was adjusted to a final $\mathrm{pH}$ of 4.5 with the addition of $\mathrm{NaOH}$, with was and filtered $(0.45 \mu \mathrm{m}$, Millipore filter, USA) prior to use. Standards were diluted in perchloric acid $(0.1 \mathrm{~N})$ in increments of 3.1, 6.2, 12.5, 25, 50, 100, 200, and 400 $\mathrm{pg} / 20 \mu \mathrm{L}$. Samples were analyzed by using the Chromelian 6.8 software program (Dionex, USA). The assay sensitivity $(6.2-12.5 \mathrm{pg} / 20 \mu \mathrm{L})$ was determined by observing reliable peaks above 
191 baseline noise. Striatal DA values were compared to DA levels in a vehicle-control group of six

192 mice (three males and three females) made up of littermates from the three cohorts of MPTP-

193 treated mice (i.e. one male and one female from each cohort).

194 Statistical analysis

195 We used a rank-based Student's $t$-test with the Welch-Satthewaite correction for

196 heteroscedasticity to compare DA loss between genders. This method was recommended by

197 Ruxton (2006) as the most robust method for two-group mean comparisons. We used linear

198 mixed-effects Analyses of Covariance (ANCOVA) to test for gender differences in the

199 longitudinal effects of MPTP toxicity on locomotor performance. The mixed-effects model

200 allowed us to incorporate all relevant fixed factors into our analyses (i.e. gender and days since

201 MPTP treatment), whilst controlling for random variation amongst the mice within each gender

202 group (Batka et al. 2014). All measures were averaged across strides within each experimental

203 day for each mouse, so that the unit of analysis was set as the average response of each mouse on

204 each day of testing. We fitted a full model for each measure, testing for 1) a main effect of

205 gender, 2) a significant association with the number of days since MPTP treatment and 3) an

206 interaction between gender and the number of days since MPTP treatment. A significant

207 interaction indicates that males and females responded differently to MPTP toxicity. In this case,

208 we report separate gender-specific regression slopes (and 95\% confidence intervals on those

209 slopes) in order to better illustrate the magnitude and direction of the interaction effect.

210 Additionally, due to the pervasive influence of locomotor speed on most measures of rodent

211 locomotor performance (Batka et al. 2014), in the event of a significant gender-specific

212 regression, we reanalyzed the relationship between the performance variable and the days since

213 MPTP treatment with speed included as a covariate in the model. In these cases, any significant 
214 residual association between a performance variable and the days since MPTP treatment

215 indicates that the indicated change in gait mechanics is not simply an after-effect of mice

216 changing their average locomotor speed. All statistical analyses were performed using the $\mathrm{R}$

217 statistical package (R Core Team 2013), including the nlme (Pinheiro et al. 2013) and lsmeans

218 (Lenth 2014) add-on packages.

\section{Results}

220

221

222

223

224

225

226

227

228

229

230

231

232

233

234

235

Striatal DA levels

Relative to control values, striatal DA levels in MPTP-treated mice decreased by an average of $54.8 \%$ amongst treated males and $58.8 \%$ amongst treated females (Fig. 2). Although striatal DA values in both genders were significantly lower than those in the vehicle control group (males: $\mathrm{t}_{[4]}=-7.8, p=0.001$; female: $\mathrm{t}_{[3]}=-7.7, p=0.005$ ), loss of striatal DA was statistically similar between genders $\left(\mathrm{t}_{[7]}=0.48, p=0.64\right)$.

\section{Locomotor performance}

A total of 668 symmetrical strides were analyzed for this study (287 strides from female mice, 381 strides from male mice). A breakdown of the number of strides coded for each experiment day and grouped by gender is provided in Table 1. Variation in the number of valid strides available for analysis on each day accounts for the unequal number of strides across the tabular cells.

Mixed-effects analyses of covariance indicated significant gender-by-days-since-MPTPtreatment interaction for all variables ( $p \leq 0.029$; results summarized in Table 2). In each case, post-hoc analysis of gender-specific regression slopes indicated that the locomotor performance of males significantly changed following MPTP administration, whereas females showed no 
236 significant response to MPTP treatment (i.e. the $95 \%$ confidence intervals about the female

237 slopes encompassed zero). Male mice responded to MPTP toxicity by decreasing speed, taking

238 shorter and fewer strides (i.e. decreasing stride length and frequency), increasing the average

239 number of supporting limbs on the ground during the stride (i.e. increasing mean support

240 number) and decreasing mediolateral sway (i.e. lower sway indices) (Table 2; Fig. 3).

241 Because speed has been shown to exert a pervasive influence on other measures of

242 locomotor performance in rodents (Batka et al. 2014), we reassessed the significant relationships

243 between the number of days following MPTP treatment and stride length, stride frequency, mean

244 support number, and sway index in the male mice, whilst controlling for speed using linear

245 mixed-effects multiple regression (results summarized in Table 3). All four variables are

246 significantly associated with speed $(p<0.001)$, suggesting that longitudinal changes in speed

247 may have affected other measures of locomotor performance. Indeed, after controlling for speed,

248 longitudinal decreases in stride length and stride frequency were found to no longer reach

249 statistical significance $(p \geq 0.415)$. In contrast, longitudinal changes in mean support number and

250 sway index remained significant even after including speed as a covariate in the model (all $p \leq$ $2510.032)$.

\section{Discussion}

253

Tests designed to economically and accurately assess gait function in PD animal models are currently lacking (but see Amende et al. 2005). Sensitive assessment tools capable of 255 capturing the different stages of PD in well-established animal models of the human condition remain a challenge to PD researchers interested in screening for and developing treatments and

257 diagnostic markers for translational use (Pienaar et al. 2012). Here we show that videography258 based measurements of gait kinematics represent a robust method to assess behavioral deficits in 
259 a well-validated mouse model of PD. In PD patients, alterations in the functioning of the

260 nigrostriatal dopaminergic system (NSDA) manifest as changes in sensorimotor functions

261 (DeLong \& Wichmann 2009). In mice, PD can be modeled to a remarkably accurate extent with

262 systematic treatment of the relatively selective NSDA neurotoxin, MPTP. A potential drawback

263 of this model is that seeing early changes in the motor behavior of mice when treated with MPTP

264 may be difficult to observe (Blandini \& Armentero 2012; Bove \& Perier 2012; Morin et al. 2014;

265 Tieu 2011). One possible explanation for this difficulty in detecting early motor changes in mice

266 is that current behavioral assays employed by PD researchers are insensitive to the subtle

267 behavioral changes shown by genetic-based or toxin-induced animal models of human PD

268 (Pienaar et al. 2012). These metrics, which typically include gait measures (e.g. stride length:

269 Fernagut et al. 2002), the pole test (i.e. time required to descend a given pole: Matsuura et al.

270 1997) for assessing bradykinesia, performance on a rotarod spindle for measuring balance, grip

271 strength and motor coordination (Rozas et al. 1997), and a balancing task, assessed by means of

272 a beam walking task (McDermott et al. 1994). However, these tests provide only a gross

273 assessment of sensorimotor function, with little distinction made between the precise functional

274 impairments. Additionally, in many of these assays, rodents are required to perform tasks that are

275 outside of their natural locomotor behavior, making it difficult to see subtle changes in function.

276 Accordingly, marked benefits could ensue with the use of novel approaches and

277 perspectives for the study of motor behavior in animal models of PD. In particular, detailed

278 analyses of gait and balance during normal rodent locomotion promise to reveal subtle changes

279 in motor behavior that may be difficult to detect using common techniques (Wang et al. 2012).

280 Moreover, such analyses have the potential to better model the bradykinesia (slowed

281 movements), festination (short, shuffling steps), postural abnormality, and gait instability that 
282 characterizes PD patients. The significance of such an approach is the prospective for identifying 283 specific sensorimotor behavioral markers of NSDA neurodegeneration. Since symptoms 284 associated with PD are not evident until advanced stages of NSDA neurodegeneration, the 285 possibility for identifying behavioral changes at an earlier stage of lesion progression in animal 286 models of PD may permit more accurate screening of novel drugs or stimulatory implants, 287 informing our understanding of the etiology of the human form of the disease and leading to 288 more promising treatments.

289 One approach for performing such sophisticated analyses of motor behavior consists of 290 the biomechanical analysis of gait dynamics. Recently, Wang and others (2012) commented that 291 gait measures specifically focusing on murine models of PD are currently lacking in the literature. 292 The precise, quantitative descriptions of animal movement presented in this study have the 293 potential to identify more subtle behavioral outputs of motor-related neurodegeneration than 294 currently available locomotor assays offer. Moreover, the robust comparative literature that 295 currently exists on the biomechanics of animal quadrupedal gait (Batka et al. 2014; Young 2012) 296 offers a solid methodological and theoretical framework from which to construct and evaluate 297 the analyses used in our research.

298 The use of the video analysis system was able to give significant insights into the gait 299 deficits, which were subtle at times, of MPTP-induced Parkinsonian mice. In male mice, but not 300 in the females, there was a significant change in gait behavior following the loss of striatal DA 301 content (Fig. 3). Using our novel experimental protocol, we were able to observe changes in the 302 gait dynamics of the mice soon after MPTP administration that correlated with the bradykinesia 303 and hyper-rigidity seen in PD patients (Hanakawa et al. 1999). Specifically, following MPTP 304 administration, male mice used slower, shorter and less frequent strides, selected footfall patterns 
305 that ensured a greater number of supporting limbs across the stride and decreased mediolateral

306 sway (Fig. 3). The gait deficits seen in the male mice became more pronounced throughout the

307 period following MPTP treatment, indicating a longitudinal decrease in locomotor performance

308 as the neurodegenerative effects of MPTP-toxicity became more pronounced (Klemann et al.

309 2015). Although loss in stride length and frequency appeared to be side effects of the general

310 decrease in locomotor speed, longitudinal changes in mean support number and sway index took

311 place independently of the changes in locomotor speed (Table 3). The longitudinal increase in

312 mean support number suggests that male mice altered their gait in some way to ensure that a

313 greater number of limbs contacted the floor surface throughout the stride, serving as a

314 compensatory effect for promoting greater gait control, to likely be an attempt at increasing

315 locomotor stability. Similarly, the longitudinal decline in the sway index may indicate a

316 decreased ability to cope with challenges to mediolateral stability, as recently demonstrated in

317 human PD patients (Galna et al. 2013). Although it is possible that some of the gait changes

318 observed during the first 1-2 days post-injection could have been associated with an acute

319 inflammatory response to MPTP, subsequent deficits in locomotor performance are best

320 explained as sequelae of neurodegeneration, mimicking the phenotypic processes that occur

321 during human PD (Klemann et al. 2015), mimicking the phenotypic processes that occur during

322 human PD.

323 Interestingly, MPTP-treated female mice showed a dramatic difference in response to the

324 MPTP toxicity. Taking all of the results into account, female mice showed a delayed and

325 diminished response to MPTP on gait function, agreeing with findings that premenopausal

326 women are at lower risk of developing Parkinsonism (Van Den Eeden et al. 2003), and that the

327 clinical phenotype of PD in women often shows a delayed presentation that is reduced in severity 
328 when compared to men (Gillies et al. 2014). Our findings are also in agreement with previous

329 studies on the gender bias in MPTP treated mice (Antzoulatos et al. 2010).

330 Although the posthumous processing of striatal tissue indicated similar percent losses of

331 dopaminergic neurons in males and females in response to MPTP, C57BL/6J females typically

332 show substantially greater absolute levels of striatal DA (Dluzen et al. 1996). Accordingly, a

333 critical absolute concentration of DA may remain in females to enable their relatively better gait

334 performance. It is also likely that the observed gender differences in gait dysfunction are due to

335 compensatory processes unique to females. Specifically, estradiol has been shown to promote

336 DA turnover, -synthesis, and -release, while simultaneously suppressing reuptake, providing a

337 sex-specific buffering mechanism for preserving behavioral function despite the depletion of

338 dopaminergic populations of neurons (Dluzen \& Horstink 2003; Gillies et al. 2014). While

339 estrogen is capable of producing neuroprotection against MPTP in both male and female

340 C57BL/6J mice (Dluzen et al. 1996), the greater endogenous levels of estrogen along with

341 greater basal striatal DA concentrations of females may contribute to such compensatory

342 processes resulting in a maintenance of female performance in our locomotor task.

\section{Conclusions}

344

Economical tests designed to accurately assess natural gait function in PD animal models are currently lacking. Here we illustrate the ability of a novel testing protocol using video-based analysis of movement to provide insight into the gait performance of MPTP-treated mice. We were able to show changes in gait function in early stages following MPTP treatment. The male group showed a statistically significant higher propensity towards gait changes than the female mice, suggesting that gait deficits in female MPTP-treated mice might be subtler. Future work should consider carrying out the described gait analyses in a cross-sectional manner, allowing for 
351 repeated assays of striatal DA content following MPTP administration to facilitate more precise

352 testing for possible associations between striatal DA depletion and locomotor dysfunction.

353 Overall, the results of our study demonstrate clearly that the novel methodology proposed here

354 has the potential to precisely quantify gait changes in animal models of PD. As such, our

355 measures could be used as output metrics in the initial screening and optimization of compounds

356 and surgical interventions for slowing, or even reversing, disease progression. 
358 We thank Bartholomew White and Michael Pante for contributing to data coding and

359 providing technical assistance. The members of the NEOMED Comparative Biomechanics

360 Journal Club provided helpful advice during the preparation of this manuscript. 
362 Amende I, Kale A, McGue S, Glazier S, Morgan JP, and Hampton TG. 2005. Gait dynamics in mouse models of Parkinson's disease and Huntingdon's disease. Journal of neuroengineering and rehabilitation 2:20.

365 366

Antony PM, Diederich NJ, and Balling R. 2011. Parkinson's disease mouse models in translational research. Mammalian Genome 22:401-419.

Antzoulatos E, Jakowec MW, Petzinger GM, and Wood RI. 2010. Sex differences in motor behavior in the MPTP mouse model of Parkinson's disease. Pharmacology, Biochemistry and Behavior 95:466-472.

Aviles-Olmos I, Limousin P, Lees A, and Foltynie T. 2013. Parkinson's disease, insulin resistance and novel agents of neuroprotection. Brain 136:374-384.

Batka RJ, Brown TJ, McMillan KP, Meadows RM, Jones KJ, and Haulcomb MM. 2014. The need for speed in rodent locomotion analyses. The Anatomical Record Part A: Discoveries in Molecular, Cellular, and Evolutionary Biology 297:1839-1864.

Bezard E, and Przedborski S. 2011. A tale on animal models of Parkinson's disease. Movement Disorders 26:993-1002.

Blandini F, and Armentero MT. 2012. Animal models of Parkinson's disease. FEBS J 279:11561166.

Bove J, and Perier C. 2012. Neurotoxin-based models of Parkinson's disease. Neuroscience 211:51-76. 
381 Bury A, and Pienaar IS. 2013. Behavioral testing regimens in genetic-based animal models of 382 Parkinson's disease: cogencies and caveats. Neuroscience and Biobehavioral Reviews 383

384 385 $37: 846-859$.

Connolly BS, and Lang AE. 2014. Pharmacological treatment of Parkinson disease: a review. JAMA : the journal of the American Medical Association 311:1670-1614.

DeLong M, and Wichmann T. 2009. Update on models of basal ganglia function and dysfunction. Parkinsonism \& Related Disorders 15:S237-S240.

Dluzen D, and Horstink M. 2003. Estrogen as neuroprotectant of nigrostriatal dopaminergic system: laboratory and clinical studies. Endocrine 21:67-75.

Dluzen DE, McDermott JL, and Liu B. 1996. Estrogen as a neuroprotectant against MPTPinduced neurotoxicity in C57/BI6 mice. Neurotoxicology and Teratology 18:603-606.

Fernagut PO, Diguet E, Labattu B, and Tison F. 2002. A simple method to measure stride length as an index of nigrostriatal dysfunction in mice. Journal of Neuroscience Methods 113:123-130.

Galna B, Murphy AT, and Morris ME. 2013. Obstacle crossing in Parkinson's disease: mediolateral sway of the centre of mass during level-ground walking and obstacle crossing. Gait and Posture 38:790-794.

Geldenhuys WJ, Kochi A, Lin L, Sutariya V, Dluzen DE, Van der Schyf CJ, and Lim MH. 2014. Methyl Yellow: A Potential Drug Scaffold for Parkinson's Disease. Chembiochem. 

Frontiers in Neuroendocrinology 35:370-384.

402

403

Hanakawa T, Katsumi Y, Fukuyama H, Honda M, Hayashi T, Kimura J, and Shibasaki H. 1999. Mechanisms underlying gait disturbance in Parkinson's disease: a single photon emission computed tomography study. Brain 122 ( Pt 7):1271-1282.

Hartmann A. 2004. Postmortem studies in Parkinson's disease. Dialogues Clin Neurosci 6:281293.

Hildebrand M. 1965. Symmetrical gaits of horses. Science 150:701-708.

Jamon M, and Clarac F. 1998. Early walking in the neonatal rat: a kinematic study. Behavioral Neuroscience 112:1218-1228.

Klemann CJHM, Martens GJM, Poelmans G, and Visser JE. 2015. Validity of the MPTP-treated mouse as a model for Parkinson's disease. Molecular Neurobiology:1-12.

Lawson RA, Yarnall AJ, Duncan GW, Khoo TK, Breen DP, Barker RA, Collerton D, Taylor JP, and Burn DJ. 2014. Severity of mild cognitive impairment in early Parkinson's disease contributes to poorer quality of life. Parkinsonism Relat Disord 20:1071-1075.

Lenth RV. 2014. 1smeans: Least-squares means. R package version 211: http://CRAN.Rproject.org/package $=1$ smeans .

Matsuura K, Kabuto H, Makino H, and Ogawa N. 1997. Pole test is a useful method for evaluating the mouse movement disorder caused by striatal dopamine depletion. Journal of Neuroscience Methods 73:45-48.

Mazzone P, Paoloni M, Mangone M, Santilli V, Insola A, Fini M, and Scarnati E. 2014. 
Unilateral deep brain stimulation of the pedunculopontine tegmental nucleus in idiopathic Parkinson's disease: effects on gait initiation and performance. Gait and Posture 40:357362.

McDermott JL, Kreutzberg JD, Liu B, and Dluzen DE. 1994. Effects of estrogen treatment on sensorimotor task performance and brain dopamine concentrations in gonadectomized male and female CD-1 mice. Hormones and Behavior 28:16-28.

Meredith GE, and Rademacher DJ. 2011. MPTP mouse models of Parkinson's disease: an update. J Parkinsons Dis 1:19-33.

Morin N, Jourdain VA, and Di Paolo T. 2014. Modeling dyskinesia in animal models of Parkinson disease. Experimental Neurology 256:105-116.

Obeso JA, Rodriguez-Oroz MC, Stamelou M, Bhatia KP, and Burn DJ. 2014. The expanding universe of disorders of the basal ganglia. Lancet 384:523-531.

Ozkaya N, and Nordin M. 1999. Fundamentals of Biomechanics: Equilibrium, Motion and Deformation. New York: Springer.

Pienaar IS, Lee CH, Elson JL, McGuinness L, Gentleman SM, Kalaria RN, and Dexter DT. 2015. Deep-brain stimulation associates with improved microvascular integrity in the subthalamic nucleus in Parkinson's disease. Neurobiology of Disease 74:392-405.

Pienaar IS, Lu B, and Schallert T. 2012. Closing the gap between clinic and cage: sensori-motor and cognitive behavioural testing regimens in neurotoxin-induced animal models of Parkinson's disease. Neuroscience and Biobehavioral Reviews 36:2305-2324. 
441 Pinheiro J, Bates D, DebRoy S, and Sarkar D. 2013. nlme: Linear and Nonlinear Mixed Effects

442 Models. R package version 31-109: http://CRAN.R-project.org/package=nlme.

443 R Core Team. 2013. R: a language and environment for statistical computing. 2.15.3 ed. Vienna, $444 \quad$ Austria: R Foundation for Statistical Computing.

445 Rozas G, Guerra MJ, and Labandeira-García JL. 1997. An automated rotarod method for quantitative drug-free evaluation of overall motor deficits in rat models of parkinsonism. Brain Research Brain Research Protocols 2:75-84.

Ruxton GD. 2006. The unequal variance t-test is an underused alternative to Student's t-test and the Mann-Whitney U-test. Behavioral Ecology 17:688-690.

Schmidt N, and Ferger B. 2001. Neurochemical findings in the MPTP model of Parkinson's disease. Journal of Neural Transmission 108:1263-1282.

Tieu K. 2011. A guide to neurotoxic animal models of Parkinson's disease. Cold Spring Harb Perspect Med 1:a009316.

Van Den Eeden SK, Tanner CM, Bernstein AL, Fross RD, Leimpeter A, Bloch DA, and Nelson LM. 2003. Incidence of Parkinson's disease: variation by age, gender, and race/ethnicity. American Journal of Epidemiology 157:1015-1022.

Walker JA. 1998. Estimating velocities and accelerations of animal locomotion: a simulation 458 experiment comparing numerical differentiation algorithms. Journal of Experimental Biology 201:981-995.

460 Wang XH, Lu G, Hu X, Tsang KS, Kwong WH, Wu FX, Meng HW, Jiang S, Liu SW, Ng HK, 

and Poon WS. 2012. Quantitative assessment of gait and neurochemical correlation in a classical murine model of Parkinson's disease. BMC Neurosci 13:142.

463 Young JW. 2012. Gait selection and the ontogeny of quadrupedal walking in squirrel monkeys 464 (Saimiri boliviensis). American Journal of Physical Anthropology 147:580-592.

465

466 
468 S1 Data. Supporting data file. The data from this study are provided in a comma469 separated (.CSV) file containing the locomotor measures and striatal dopamine levels for each 470 mouse in the dataset. Note that dopamine levels were evaluated relative to a control value of $471 \quad 19,037$ obtained by assaying the striatal tissue of six non-treated mice. 
473 Figure 1. Sample frame from high-speed video analyses of mouse locomotion. Video analysis 474 software was used to digitize the two-dimensional position of the nose and tail base during the 475 pass down the trackway. A pair of mirrors angled at $45^{\circ}$ to the sagittal plane allowed for 476 capturing touchdown and lift-off events over complete gait cycles.

477

478 Figure 2. Measures of striatal DA levels in male and female mice. Each bar represents mean \pm 479 S.D. $(\mathrm{N}$ females $=4, \mathrm{~N}$ males $=5)$. Asterisks indicate that $\mathrm{DA}$ levels in treated males and females 480 were significantly lower than in the vehicle-control group ( $p<0.01$ for both genders).

481

482 Figure 3. Longitudinal changes in locomotor performance following MPTP administration in 483 male and female mice. Panels show the individual mean values of (a) locomotor speed, (b) stride 484 length, (c) stride frequency, (d) mean support number and (e) sway index at baseline (BL) and at 485 each day following MPTP treatment. Trend lines indicate gender-specific linear mixed-effects 486 regression slopes.

487

488

489

490 


\section{Table $\mathbf{1}$ (on next page)}

Tables 


\section{TABLES}

Table 1. Sample sizes of locomotor strides, grouped by gender and MPTP treatment day.

\begin{tabular}{ccccccccc}
\hline & \multicolumn{9}{c}{ MPTP Treatment Day } \\
& Baseline & Day 1 & Day 2 & Day 3 & Day 4 & Day 5 & Day 6 & Day 7 \\
\hline Female & 56 & 40 & 28 & 23 & 24 & 39 & 35 & 42 \\
Male & 68 & 43 & 30 & 29 & 39 & 53 & 56 & 64 \\
\hline
\end{tabular}

3 
4 Table 2. Analyses of covariance of locomotor performance variables by gender and MPTP

5 treatment day.

6

\begin{tabular}{|c|c|c|c|}
\hline Variable & Interaction $^{\text {Effect }}{ }^{\mathbf{1}}$ & & $\begin{array}{c}\text { Slope } \\
{[95 \% \text { confidence interval }]^{2}}\end{array}$ \\
\hline Speed & $\begin{array}{c}-1.87 \\
\mathrm{~F}_{[1,43]}=7.93 \\
p=\mathbf{0 . 0 0 7}\end{array}$ & $\begin{array}{c}\text { Female slope: } \\
\text { Male slope: }\end{array}$ & $\begin{array}{c}0.616 \\
{[-0.373,1.61]} \\
-1.25 \\
{[-2.15,-0.351]}\end{array}$ \\
\hline Stride length & $\begin{array}{c}-0.0998 \\
\mathrm{~F}_{[1,43]}=7.69 \\
p=\mathbf{0 . 0 0 8}\end{array}$ & $\begin{array}{c}\text { Female slope: } \\
\text { Male slope: }\end{array}$ & $\begin{array}{c}0.0365 \\
{[-0.0173,0.0902]} \\
-0.0633 \\
{[-0.112,-0.0145]}\end{array}$ \\
\hline Stride frequency & $\begin{array}{c}-0.136 \\
\mathrm{~F}_{[1,43]}=7.05 \\
p=\mathbf{0 . 0 1 1}\end{array}$ & $\begin{array}{c}\text { Female slope: } \\
\text { Male slope: }\end{array}$ & $\begin{array}{c}0.0412 \\
{[-0.0351,0.118]} \\
-0.0945 \\
{[-0.164,-0.0252]}\end{array}$ \\
\hline $\begin{array}{l}\text { Mean support } \\
\text { number }\end{array}$ & $\begin{array}{c}0.0254 \\
\mathrm{~F}_{[1,43]}=5.07 \\
p=\mathbf{0 . 0 3 0}\end{array}$ & $\begin{array}{c}\text { Female slope: } \\
\text { Male slope: }\end{array}$ & $\begin{array}{c}0.00355 \\
{[-0.0133,0.0204]} \\
0.0290 \\
{[0.0136,0.0443]}\end{array}$ \\
\hline Sway index & $\begin{array}{c}-0.0539 \\
\mathrm{~F}_{[1,43]}=8.5 \\
p=\mathbf{0 . 0 0 6}\end{array}$ & Female slope: & $\begin{array}{c}0.00996 \\
{[-0.0176,0.0375]} \\
-0.0439 \\
{[-0.0689,-0.0189]}\end{array}$ \\
\hline
\end{tabular}

\footnotetext{
${ }^{1}$ The interaction effect indicates the average difference in slope between male and female mice (i.e., male slope - female slope), and the significance of this difference relative to the null expectation of zero. Significant interactions are indicated by a $p$-value in printed in bold type.

2 These values indicate the gender specific slope of the performance variable against MPTP treatment day. 95\% confidence intervals are displayed as [lower confidence bound, upper confidence bound]. Confidence bounds of opposite sign indicate a non-significant slope.
} 
15 Table 3. Association between locomotor performance variables and MPTP treatment day,

16 controlling for speed.

17

18

\begin{tabular}{cc}
\hline Variable & $\begin{array}{c}\text { Partial regression coefficient } \\
{[\mathbf{9 5 \%} \text { confidence interval }]^{1}}\end{array}$ \\
\hline Stride length & -0.016 \\
& {$[-0.0554,0.0237]$} \\
$p$ & $=0.415$ \\
Stride frequency & -0.002 \\
& {$[-0.0254,0.0209]$} \\
& $p=0.842$ \\
Mean support number & 0.010 \\
& {$[0.0001,0.0196]$} \\
& $p=\mathbf{0 . 0 3 2}$ \\
Sway index & -0.012 \\
& {$[-0.0214,-0.0018]$} \\
& $p=\mathbf{0 . 0 2 2}$ \\
\hline
\end{tabular}

19

${ }^{1}$ The partial regression coefficients reflect the residual influence of MPTP treatment day on each locomotor performance variable, controlling for the effects of speed. Significant partial regressions are indicated by a $p$-value in printed in bold type. 
1

Figure 1. Sample frame from high-speed video analyses of mouse locomotion.

Video analysis software was used to digitize the two-dimensional position of the nose and tail base during the pass down the trackway. A pair of mirrors angled at $45^{\circ}$ to the sagittal plane allowed for capturing touchdown and lift-off events over complete gait cycles.

Mirror (angled at 45 degrees)

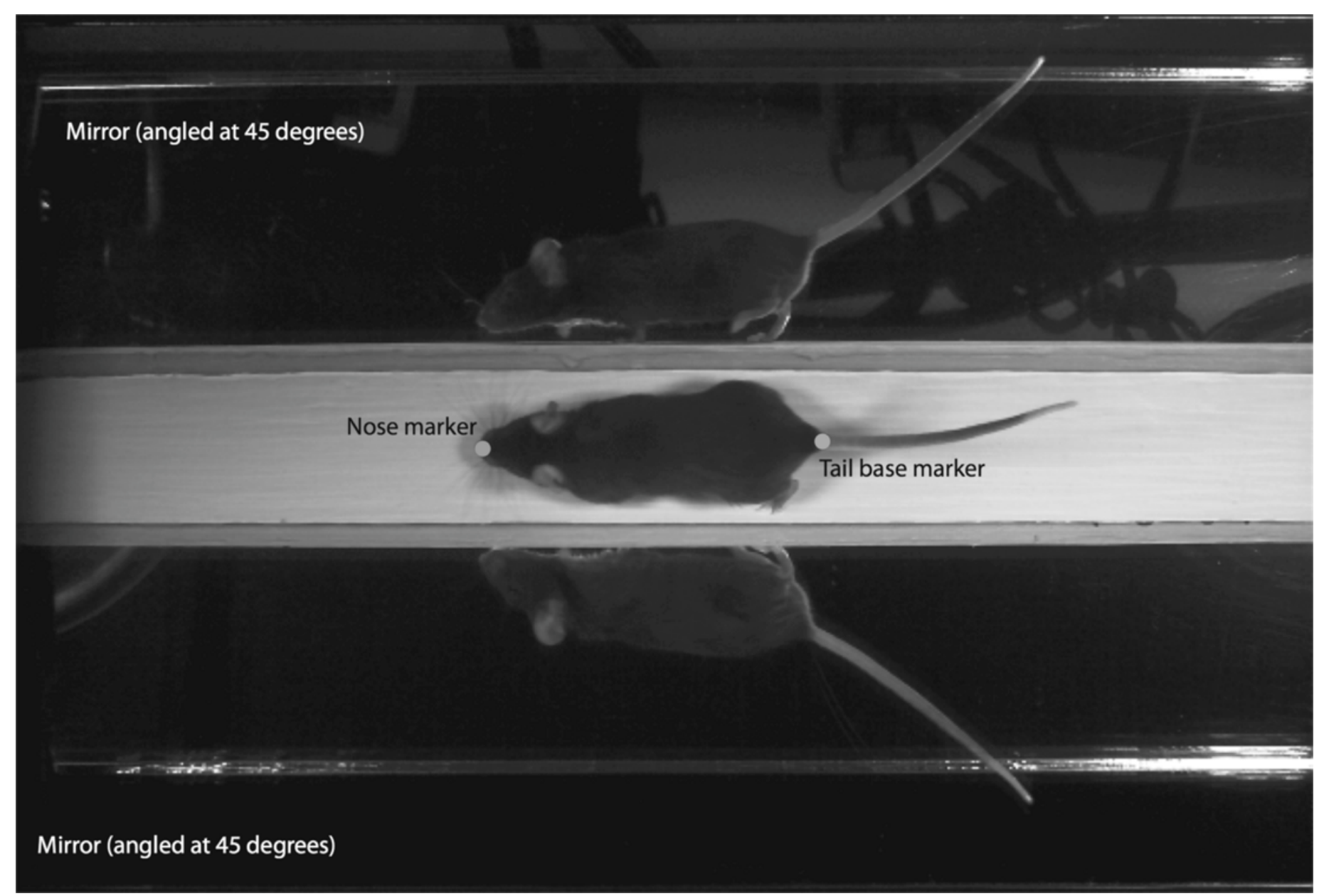


2

Figure 2. Measures of striatal DA levels in male and female mice.

Each bar represents mean \pm S.D. $(\mathrm{N}$ females $=4, \mathrm{~N}$ males $=5)$. Asterisks indicate that $\mathrm{DA}$ levels in treated males and females were significantly lower than in the vehicle-control group ( $p<0.01$ for both genders).

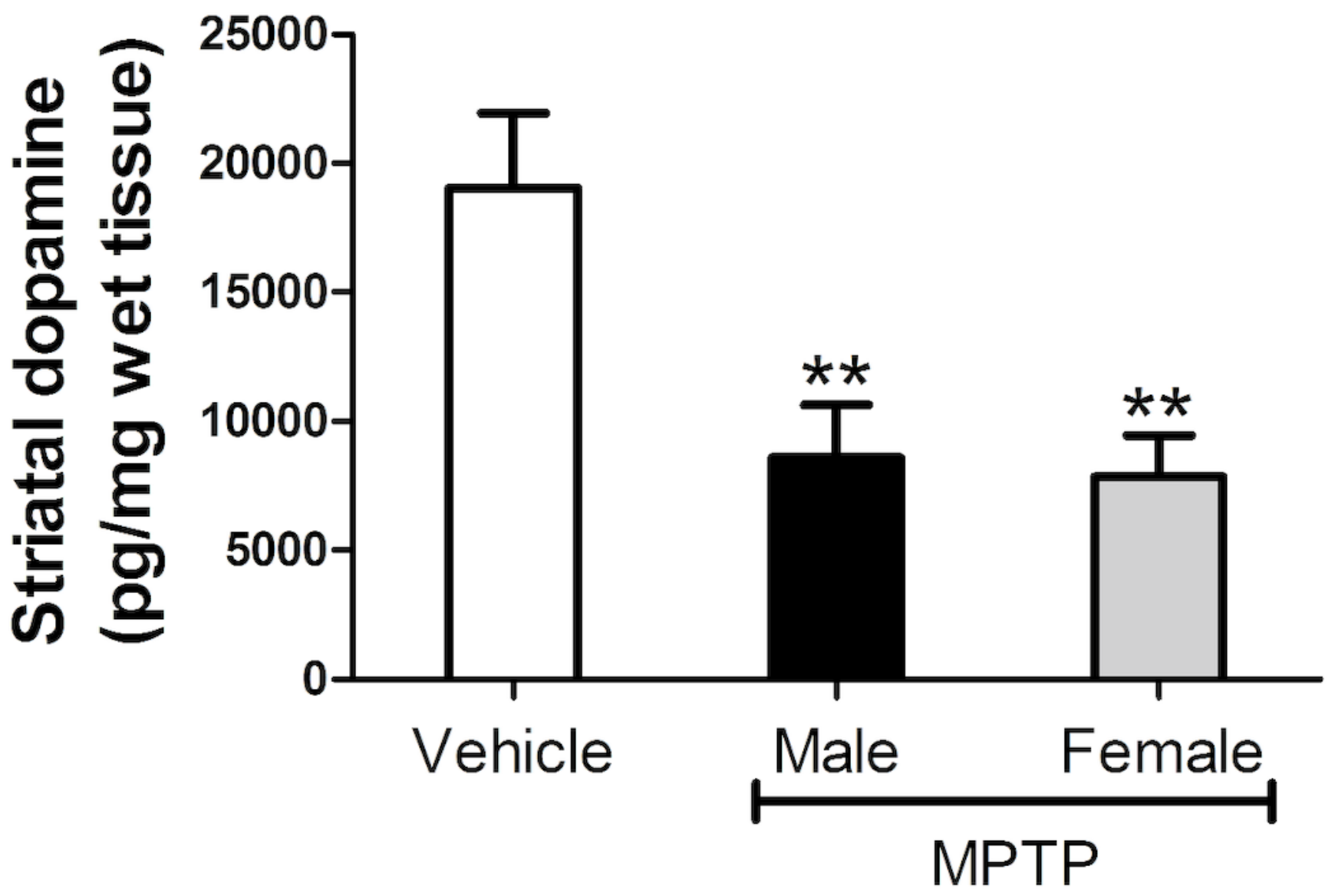


Figure 3. Longitudinal changes in locomotor performance following MPTP administration in male and female mice.

Panels show the individual mean values of (a) speed, (b) stride length, (c) stride frequency, (d) mean support number and (e) sway index at baseline (BL) at each day following MPTP treatment. Trend lines indicate gender-specific linear mixed-effects regression slopes.

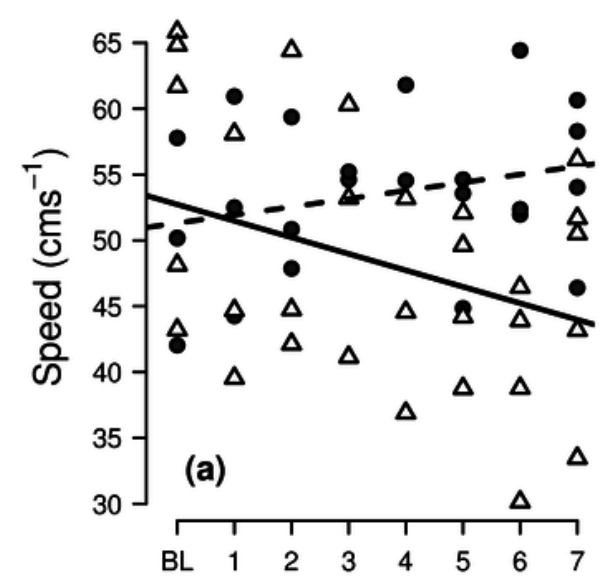

Days since MPTP treatment

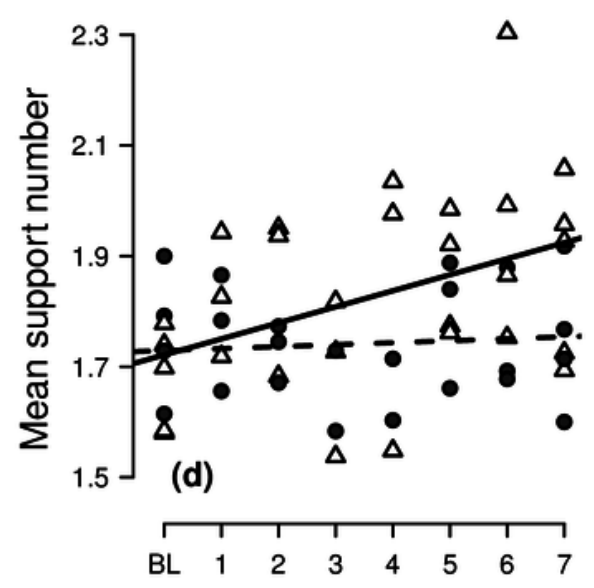

Days since MPTP treatment

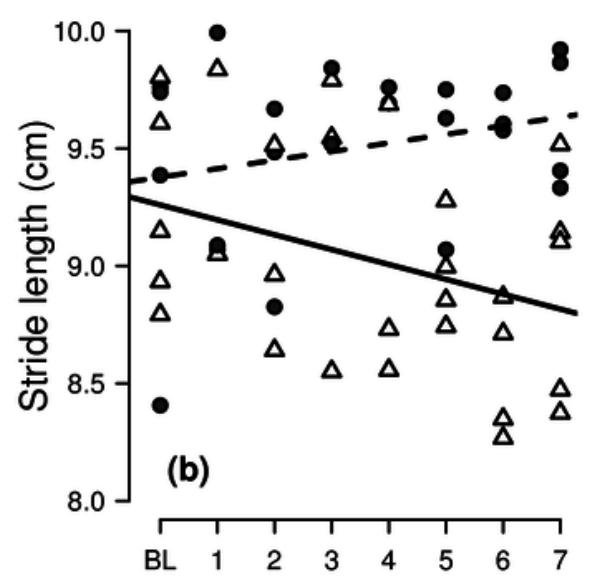

Days since MPTP treatment

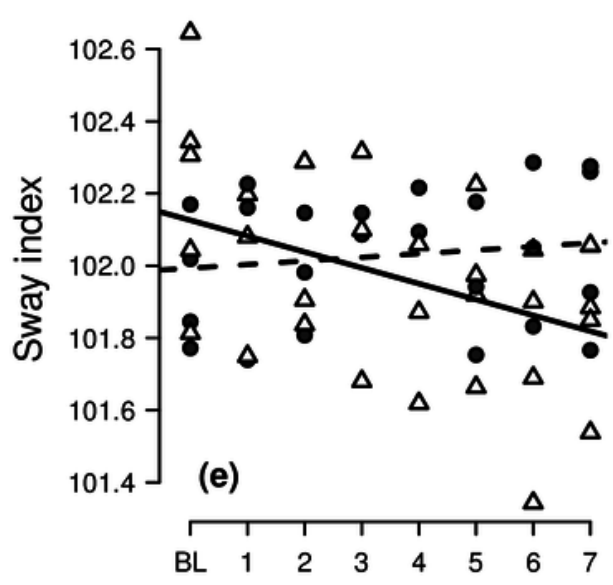

Days since MPTP treatment

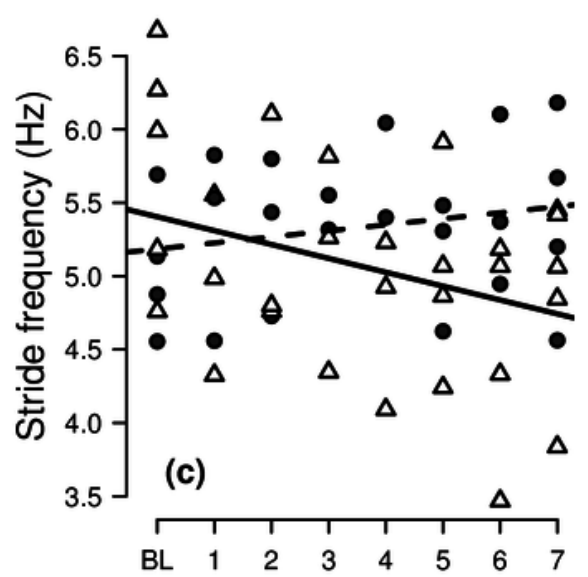

Days since MPTP treatment

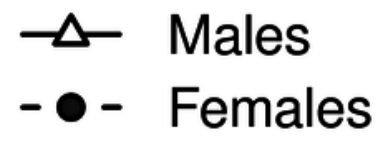

- ๑ - Females 